Diego Cedrim Gomes Rêgo

\title{
Execução Otimizada de Transações \\ Financeiras: Um Estudo Empírico
}

Dissertação apresentada como requisito parcial para obtenção do grau de Mestre pelo Programa de Pós-graduação em Informática do Departamento de Informática da PUC-Rio

Orientador: Prof. Eduardo Sany Laber 


\title{
Diego Cedrim Gomes Rêgo
}

\section{Execução Otimizada de Transações \\ Financeiras: Um Estudo Empírico}

\begin{abstract}
Dissertação apresentada como requisito parcial para obtenção do grau de Mestre pelo Programa de Pós-graduação em Informática do Departamento de Informática do Centro Técnico Científico da PUC-Rio. Aprovada pela Comissão Examinadora abaixo assinada.
\end{abstract}

\author{
Prof. Eduardo Sany Laber \\ Orientador \\ Departamento de Informática - PUC-Rio
}

Prof. Edward Hermann Haeusler

Departamento de Informática - PUC-Rio

Prof. Alexandre Roberto Renteria

PUC-Rio

Prof. José Eugenio Leal

Coordenador Setorial do Centro Técnico Científico - PUC-Rio 
Todos os direitos reservados. É proibida a reprodução total ou parcial do trabalho sem autorização da universidade, do autor e do orientador.

\section{Diego Cedrim Gomes Rêgo}

Graduou-se em Ciências da Computação na Universidade Federal de Alagoas.

Ficha Catalográfica

Rêgo, Diego Cedrim Gomes

Execução otimizada de transações financeiras: um estudo empírico / Diego Cedrim Gomes Rêgo ; orientador: Eduardo Sany Laber. - 2009.

107 f. ; $30 \mathrm{~cm}$

Dissertação (Mestrado em Informática) - Pontifícia Universidade Católica do Rio de Janeiro, Rio de Janeiro, 2009.

Inclui bibliografia.

1. Informática - Teses. 2. Aprendizado de Máquina. 3. Aprendizado por Reforço. 4. Mercados Financeiros. 5. Algoritmos. 6. Otimização. I. Laber, Eduardo. II. Pontifícia Universidade Católica do Rio de Janeiro. Departamento de Informática. III. Título. 


\section{Agradecimentos}

A Deus, por tudo.

A minha querida família em Maceió-AL e em Recife-PE.

Ao meu orientador, Eduardo Laber.

Ao Departamento de Informática da PUC-Rio.

Aos meus amigos da PUC-Rio, que foram muito importantes e tornaram esses dois anos mais agradáveis.

À CAPES e à PUC-Rio, pelos auxílios concedidos, sem os quais este trabalho não poderia ter sido realizado. 


\section{Resumo}

Rêgo, Diego Cedrim Gomes; Laber, Eduardo. Execução Otimizada de Transações Financeiras: Um Estudo Empírico. Rio de Janeiro, 2009. 107p. Dissertação de Mestrado - Departamento de Informática, Pontifícia Universidade Católica do Rio de Janeiro.

Apresentamos um estudo empírico comparativo para o problema de Execução Otimizada de Transações nos mercados financeiros modernos. Construímos um simulador dos mercados financeiros, e então, baseado nessa ferramenta, comparamos o desempenho de algumas estratégias propostas na literatura. Os melhores resultados foram obtidos por estratégias que usam técnicas de aprendizado de máquina.

\section{Palavras-chave}

Aprendizado de Máquina. Aprendizado por Reforço. Mercados Financeiros. Algoritmos. Otimização. 


\section{Abstract}

Rêgo, Diego Cedrim Gomes; Laber, Eduardo. Optimized Financial Trade Execution: A empirical study. Rio de Janeiro, 2009. 107p. MsC Dissertation - Departamento de Informática, Pontifícia Universidade Católica do Rio de Janeiro.

We present a comparative empirical study for the Optimized Trade Execution problem in moderns financial markets. We build a financial market simulator and then, based on this tool, we compare the performance of many strategies available in the literature. The best results were achieved by strategies that make use of machine learning techniques.

\section{Keywords}

Machine Learning. Reinforcement Learning. Financial Markets. Algorithms. Optimization. 


\section{Sumário}

1 Introdução 10

$\begin{array}{lll}1.1 & \text { Definição do Problema } & 10\end{array}$

$\begin{array}{lll}1.2 & \text { Contribuições } & 10\end{array}$

1.3 Trabalhos Relacionados 11

$\begin{array}{lll}1.4 & \text { Organização } & 14\end{array}$

2 Conceitos Básicos $\quad 15$

2.1 Microestrutura do Mercado $\quad 15$

$\begin{array}{ll}2.2 & \text { Aprendizado por Reforço } \\ 2.3 & 17\end{array}$

2.3 Validação de Algoritmos de Aprendizado de Máquina 26

3 Algoritmos Implementados $\quad 30$

3.1 Considerações Iniciais 30

3.2 Estratégia Submit and Execute 31

3.3 Estratégia Submit and Leave 31

3.4 Estratégia Reinforcement Learning 32

$\begin{array}{lll}3.5 & \text { Estratégia Kearns } & 39\end{array}$

3.6 Estratégia Aleatória 44

3.7 Estratégia Ótima 45

4 Análise Experimental $\quad 46$

4.1 Ambiente e Dados 46

4.2 Medição de Performance 49

4.3 Estratégias e Parâmetros 50

4.4 Resultados dos Experimentos $\quad 52$

4.5 Observações Gerais sobre os Experimentos 52

5 Conclusões e Trabalhos Futuros $\quad 65$

$\begin{array}{ll}\text { Referências Bibliográficas } & 67\end{array}$

$\begin{array}{lll}\text { A Tabelas de Resultados dos Experimentos } & \mathbf{7 0}\end{array}$ 


\section{Lista de figuras}

2.1 Modelo padrão de aprendizado por reforço 18

2.2 Labirinto 24

2.3 Política Ótima para Resolução do Labirinto 26

2.4 Método holdout 28

2.5 Seleção Aleatória de Subconjuntos (com $K=8)$

2.6 K-Fold Cross validation (com $K=4$ ) 29

2.7 Leave-one-out Cross Validation 29

3.1 Exemplo da Estratégia Submit and Execute (com $R=5)$

3.2 Estratégia Submit and Leave: Duas Ofertas (com $R=5)$

3.3 Estratégia Submit and Leave: Única Oferta (com $R=5$ ) 32

3.4 Exemplo de Flutuação do Valor de Negociação de uma Ação 34

3.5 Exemplo de Recompensa 36

3.6 Exemplo da Estratégia Reinforcement Learning 39

3.7 Curva de Retornos 41

3.8 Curva de Riscos 42

3.9 Trade-off entre risco e retorno 43

3.10 Exemplo da Estratégia Aleatória 45

4.1 Exemplo dos Dados Colhidos 48

4.2 Exemplo de Demarcação de Intervalos 49

4.3 Política Kearns (agressividade $=1.0, R=60$ e objetivo de compra) 55

4.4 Política Reinforcement Learning ( $R=60$, objetivo de compra) 56

4.5 Retornos recebidos em função do fator (Compra) 57

4.6 Retornos recebidos em função do fator (Venda) 58 


\section{Lista de tabelas}

2.1 Book de Compra 16

2.2 Book de Venda 16

2.3 Exemplo de diálogo entre o agente e o ambiente 19

2.4 Sequência de aprendizado da política ótima 26

3.1 Atributos Privados 33

3.2 Atributos Públicos 33

3.3 Espaço de Ações do Agente 35

3.4 Exemplo de Possíveis Ações em Estados 36

3.5 Exemplo de Política de Venda 39

4.1 Lista de Experimentos Executados 53

4.2 Pontuação Geral das Estratégias 61

4.3 Pontuação das Estratégias (Venda) 63

4.4 Pontuação das Estratégias (Compra) 64

A.1 10-Fold Cross Validation, $R=10$, Compra, Dólar 71

A.2 10-Fold Cross Validation, $R=30$, Compra, Dólar 73

A.3 10-Fold Cross Validation, $R=60$, Compra, Dólar 74

A.4 Método Holdout, $R=10$, Compra, Dólar 76

A.5 Método Holdout, $R=30$, Compra, Dólar 78

A.6 Método Holdout, $R=60$, Compra, Dólar $\quad 79$

A.7 Método Holdout, $R=10$, Venda, Dólar $\quad 81$

A.8 Método Holdout, $R=30$, Venda, Dólar $\quad 82$

A.9 Método Holdout, $R=60$, Venda, Dólar 84

A.10 10-Fold Cross Validation, $R=10$, Venda, Dólar 85

A.11 10-Fold Cross Validation, $R=30$, Venda, Dólar 87

A.12 10-Fold Cross Validation, $R=60$, Venda, Dólar 88

A.13 10-Fold Cross Validation, $R=10$, Compra, Petrobras 90

A.14 10-Fold Cross Validation, $R=30$, Compra, Petrobras 91

A.15 10-Fold Cross Validation, $R=60$, Compra, Petrobras 93

A.16 10-Fold Cross Validation, $R=10$, Venda, Petrobras 95

A.17 10-Fold Cross Validation, $R=30$, Venda, Petrobras 96

A.18 10-Fold Cross Validation, $R=60$, Venda, Petrobras 98

A.19 10-Fold Cross Validation, $R=10$, Compra, Vale 99

A.20 10-Fold Cross Validation, $R=30$, Compra, Vale 101

A.21 10-Fold Cross Validation, $R=60$, Compra, Vale 102

A.22 10-Fold Cross Validation, $R=10$, Venda, Vale 104

A.23 10-Fold Cross Validation, $R=30$, Venda, Vale 105

A.24 10-Fold Cross Validation, $R=60$, Venda, Vale 107 